\title{
Landau-Zener Treatments of Solar Neutrino Oscillations
}

\author{
Marcus Bruggen, $a, b$ W.C. Haxton, $b$ and Y.-Z. Qian $b$ \\ aDepartment of Physics, Cambridge University, Cambridge CB3 OHE, United Kingdom \\ bInstitute for Nuclear Theory, NK-12, and Department of Physics, NK-15, University of Washington, \\ Seattle, WA 98195 USA
}

\section{PREPARED FOR THE U.S. DEPARTMENT OF ENERGY UNDER GRANT DE-FG06-90ER40561}

This report was prepared as an account of work sponsored by the United States Government. Neither the United States nor any agency thereof, nor any of their employees, makes any warranty, express or implied, or assumes any legal liability or responsibility for the accuracy, completeness, or usefulness of any information, apparatus, product, or process disclosed, or represents that its use would not infringe privately owned rights. Reference herein to any specific commercial product, process, or service by trade name, mark, manufacturer, or otherwise, does not necessarily constitute or imply its endorsement, recommendation, or favoring by the United States Government or any agency thereof. The views and opinions of authors expressed herein do not necessarily state or reflect those of the United States Government or any agency thereof.

\section{MASTER}

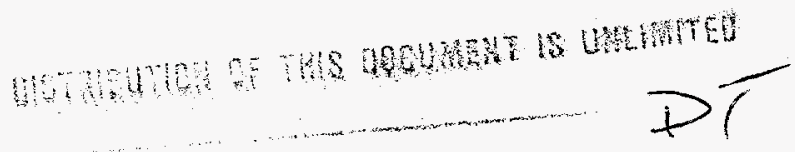




\section{DISCLAIMER}

This report was prepared as an account of work sponsored by an agency of the United States Government. Neither the United States Government nor any agency thereof, nor any of their employees, make any warranty, express or implied, or assumes any legal liability or responsibility for the accuracy, completeness, or usefulness of any information, apparatus, product, or process disclosed, or represents that its use would not infringe privately owned rights. Reference herein to any specific commercial product, process, or service by trade name, trademark, manufacturer, or otherwise does not necessarily constitute or imply its endorsement, recommendation, or favoring by the United States Government or any agency thereof. The views and opinions of authors expressed herein do not necessarily state or reflect those of the United States Government or any agency thereof. 


\section{DISCLAIMER}

Portions of this document may be illegible in electronic image products. Images are produced from the best available original document. 


\title{
Landau-Zener Treatments of Solar Neutrino Oscillations
}

\author{
Marcus Bruggen, ${ }^{a, b}$ W.C. Haxton, ${ }^{b}$ and Y.-Z. Qian ${ }^{b}$ \\ ${ }^{a}$ Department of Physics, Cambridge University, Cambridge CB3 OHE, United Kingdom \\ ${ }^{b}$ Institute for Nuclear Theory, NK-12, and Department of Physics, NK-15 \\ University of Washington, Seattle, WA 98195
}

\begin{abstract}
We present a simple derivation of Landau-Zener formulae for nonadiabatic level crossings for linear and exponential density profiles. The derivation depends on a single assumption that applies somewhat differently to the exponential and linear cases, providing some insight into the relationship between the respective formulae. We note that one exponential Landau-Zener formula in common use is only approximately correct. Comparisons with exact calculations are made.
\end{abstract}


Landau-Zener oscillation formulae $\mathrm{e}^{[1-6]}$ were introduced into solar neutrino calculations shortly after Mikheyev and Smirnov ${ }^{[7]}$ discovered matter-enhanced oscillations. Their importance derives from their simplicity and accuracy, as direct numerical integrations of the Mikheyev-Smirnov-Wolfenstein equations can be tedious when the full spectrum of solar neutrinos and finite extent of the solar core are taken into account.

While the correct Landau-Zener results for both linear and exponential densities can be found in the literature, the derivations of these results generally obscure the simple physical arguments underlying their validity. The purpose of this paper is largely pedagogical, to present a derivation that clearly identifies the one necessary approximation. As this approximation applies differently to the linear and exponential cases, it provides some insight into the relationship between the respective formulae. While we conclude that the exponential form offers advantages in the large mixing angle region, one commonly used exponential Landau-Zener formula is shown to be unreliable in this limit.

We begin by reviewing the two-flavor oscillation problem. Consider the case of two flavor eigenstates related to the mass eigenstates through a vacuum mixing angle $\theta_{v}$,

$$
\begin{aligned}
& \left|\nu_{e}\right\rangle=\cos \theta_{v}\left|\nu_{1}\right\rangle+\sin \theta_{v}\left|\nu_{2}\right\rangle \\
& \left|\nu_{\mu}\right\rangle=-\sin \theta_{v}\left|\nu_{1}\right\rangle+\cos \theta_{v}\left|\nu_{2}\right\rangle .
\end{aligned}
$$

If one writes the neutrino wave function in matter as

$$
|\nu(x)\rangle=a_{e}(x)\left|\nu_{e}\right\rangle+a_{\mu}(x)\left|\nu_{\mu}\right\rangle
$$

where $x$ is the coordinate along the neutrino's path. the evolution of $a_{e}(x)$ and $a_{\mu}(x)$ is governed by

$$
i \frac{d}{d x}\left(\begin{array}{c}
a_{e} \\
a_{\mu}
\end{array}\right)=\frac{1}{4 E}\left(\begin{array}{rr}
2 E \sqrt{2} G_{F} \rho(x)-\delta m^{2} \cos 2 \theta_{v} & \delta m^{2} \sin 2 \theta_{v} \\
\delta m^{2} \sin 2 \theta_{v} & -2 E \sqrt{2} G_{F} \rho(x)+\delta m^{2} \cos 2 \theta_{v}
\end{array}\right)\left(\begin{array}{l}
a_{e} \\
a_{\mu}
\end{array}\right)
$$

where $\delta m^{2}=m_{2}^{2}-m_{1}^{2}$ and $\rho(x)$ is the solar density of electrons, the variable that determines the difference in the $\nu_{e}$ and $\nu_{\mu}$ effective masses. It is convenient to rewrite Eq. (1) in a basis consisting of the light and heavy local mass eigenstates (i.e., the states that diagonalize the right-hand side of Eq. (1))

$$
\begin{aligned}
\left|\nu_{L}(x)\right\rangle & =\cos \theta(x)\left|\nu_{e}\right\rangle-\sin \theta(x)\left|\nu_{\mu}\right\rangle \\
\left|\nu_{H}(x)\right\rangle & =\sin \theta(x)\left|\nu_{e}\right\rangle+\cos \theta(x)\left|\nu_{\mu}\right\rangle .
\end{aligned}
$$

The local mixing angle is defined by

$$
\sin 2 \theta(x)=\frac{\sin 2 \theta_{v}}{\sqrt{X^{2}(x)+\sin ^{2} 2 \theta_{v}}}
$$




$$
\cos 2 \theta(x)=\frac{-X(x)}{\sqrt{X^{2}(x)+\sin ^{2} 2 \theta_{v}}}
$$

where $X(x)=2 \sqrt{2} G_{F} \rho(x) E / \delta m^{2}-\cos 2 \theta_{v}$. Thus $\theta(x)$ ranges from $\theta_{v}$ to $\pi / 2$ as the density $\rho(x)$ goes from 0 to $\infty$.

If we define

$$
|\nu(x)\rangle=a_{H}(x)\left|\nu_{H}(x)\right\rangle+a_{L}(x)\left|\nu_{L}(x)\right\rangle
$$

Eq.(1) becomes

$$
i \frac{d}{d x}\left(\begin{array}{c}
a_{H} \\
a_{L}
\end{array}\right)=\left(\begin{array}{cc}
\lambda(x) & i \alpha(x) \\
-i \alpha(x) & -\lambda(x)
\end{array}\right)\left(\begin{array}{c}
a_{H} \\
a_{L}
\end{array}\right)
$$

with the local mass eigenstate splitting determined by

$$
4 E \lambda(x)=\delta m^{2} \sqrt{X^{2}(x)+\sin ^{2} 2 \theta_{v}}
$$

and with

$$
\alpha(x)=\left(\frac{E}{\delta m^{2}}\right) \frac{\sqrt{2} G_{F} \frac{d}{d x} \rho(x) \sin 2 \theta_{v}}{X^{2}(x)+\sin ^{2} 2 \theta_{v}} .
$$

Note that the local mass eigenstate splitting achieves its minimum value, $\delta m^{2} \sin 2 \theta_{v}$, at a critical density $\rho_{c}=\rho\left(x_{c}\right)$

$$
2 \sqrt{2} E G_{F} \rho_{c}=\delta m^{2} \cos 2 \theta_{v}
$$

that defines the point where the diagonal elements of Eq. (1) cross.

Equation (3) can be trivially integrated if the splitting of the diagonal elements is large compared to the off-diagonal elements,

$$
\gamma(x)=\left|\frac{\lambda(x)}{\alpha(x)}\right|=\frac{\sin ^{2} 2 \theta_{v}}{\cos 2 \theta_{v}} \frac{\delta m^{2}}{2 E} \frac{1}{\left|\frac{1}{\rho_{c}} \frac{d \rho(x)}{d x}\right|} \frac{\left[X(x)^{2}+\sin ^{2} 2 \theta_{v}\right]^{3 / 2}}{\sin ^{3} 2 \theta_{v}} \gg 1
$$

a condition that becomes particularly stringent near the crossing point

$$
\gamma_{c}=\gamma\left(x_{c}\right)=\frac{\sin ^{2} 2 \theta_{v}}{\cos 2 \theta_{v}} \frac{\delta m^{2}}{2 E} \frac{1}{\left|\frac{1}{\rho_{c}} \frac{d \rho(x)}{d x}\right|_{x=x_{c}} \mid} \gg 1
$$

The resulting adiabatic electron neutrino survival probability, valid when $\gamma_{c} \gg 1$, is

$$
P_{\nu_{e}}^{\text {adiab }}=\frac{1}{2}+\frac{1}{2} \cos 2 \theta_{v} \cos 2 \theta_{i}
$$

where $\theta_{i}=\theta\left(x_{i}\right)$ is the local mixing angle at the density where the neutrino was produced. Eq. (6) was first discussed by Bethe. ${ }^{[8]}$ 
The above discussion immediately suggests a strategy ${ }^{[9]}$ for analytically treating nonadiabatic level crossings, $\gamma_{c} \lesssim 1$. The solar density can be replaced by a simpler, two-parameter form that is constrained to reproduce the true density and its derivative at the crossing point $x_{c}$. Two convenient choices are the linear $(\rho(x)=a+b x)$ and exponential $\left(\rho(x)=a e^{-b x}\right)$ profiles. As the density derivative at $x_{c}$ governs the nonadiabatic behavior, this procedure should provide an accurate description of the hopping probability between the local mass eigenstates when the neutrino traverses the crossing point. The initial and ending points $x_{i}$ and $x_{f}$ for the artificial profile are then chosen so that $\rho\left(x_{i}\right)$ is the density where the neutrino was produced in the solar core and $\rho\left(x_{f}\right)=0$ (the solar surface), as illustrated in Fig. 1. Since the adiabatic result (Eq. (6)) depends only on the local mixing angles at these points, this builds in that limit. Eq. (1) can then be integrated exactly for linear and exponential profiles, with the results given in terms of parabolic cylinder and Whittaker functions, respectively. This treatment, called the finite Landau-Zener approximation, ${ }^{[10,6]}$ has been used extensively in numerical calculations.

We derive a simpler ("infinite") Landau-Zener approximation ${ }^{[11]}$ by observing that the nonadiabatic region is generally confined to a narrow region around $x_{c}$, away from the endpoints $x_{i}$ and $x_{f}$. We can then extend the artificial profile to $x= \pm \infty$, as illustrated by the dashed lines in Fig. 1. As the neutrino propagates adiabatically in the unphysical region $x<x_{i}$, the exact solution in the physical region can be recovered by choosing the initial boundary conditions

$$
\begin{aligned}
& a_{L}(-\infty)=-a_{\mu}(-\infty)=\cos \theta_{i} e^{-i \int_{-\infty}^{x_{i}} \lambda(x) d x} \\
& a_{H}(-\infty)=a_{e}(-\infty)=\sin \theta_{i} e^{i \int_{-\infty}^{x_{i}} \lambda(x) d x}
\end{aligned}
$$

That is $|\nu(-\infty)\rangle$ will then adiabatically evolve to $\left|\nu\left(x_{i}\right)\right\rangle=\left|\nu_{e}\right\rangle$ as $x$ goes from $-\infty$ to $x_{i}$.

We want to express the electron neutrino survival probability as the neutrino exits the sun, $\rho\left(x_{f}\right)=0$, in terms of the asymptotic wave function $|\nu(\infty)\rangle$. In the exponential case this is trivial since the physical ending density is achieved at $x=\infty$,

$$
P_{\nu_{e}}^{\exp }=\left|\left\langle\nu_{e} \mid \nu(\infty)\right\rangle\right|^{2} .
$$

In the linear case, we must account for the (assumed adiabatic) propagation from $x_{f}$ to $\infty$ to express $P_{\nu_{e}}$ in terms of the asymptotic wave function. We find

$$
P_{\nu_{e}}^{\operatorname{lin}}=\left|\left\langle\nu_{e} \mid \nu\left(x_{f}\right)\right\rangle\right|^{2}=\left|\left\langle\nu^{\prime} \mid \nu(\infty)\right\rangle\right|^{2}
$$


where

$$
\left|\nu^{\prime}\right\rangle=\cos \theta_{v} e^{i \int_{x_{f}}^{\infty} \lambda(x) d x}\left|\nu_{e}\right\rangle+\sin \theta_{v} e^{-i \int_{x_{f}}^{\infty} \lambda(x) d x}\left|\nu_{\mu}\right\rangle
$$

Eqs. (7-9) greatly simplify our task by allowing us to solve a problem on an infinite interval, so that the asymptotic forms of the confluent hypergeometric functions can be used. In the linear case this yields ${ }^{[10]}$

$$
a_{e}(\infty)=a_{e}(-\infty) e^{-\pi \gamma_{c / 4}}-a_{\mu}(-\infty) \sqrt{\frac{\pi \gamma_{c}}{2}} \frac{e^{-\pi \gamma_{c} / 8}}{\Gamma\left(1+\frac{i \gamma_{c}}{4}\right)} e^{\frac{i \pi}{4}+\frac{i R^{2}}{2}} R^{i \gamma_{c / 2}}
$$

where $R=\lim _{x \rightarrow \infty} x \sqrt{\sqrt{2} G_{F}\left|\frac{d}{d x} \rho\left(x_{c}\right)\right|}$. The rapidly varying $\mathrm{R}$-dependent adiabatic phases average out in $P_{\nu_{e}}$ whenever one integrates over a neutrino-producing region of finite extent or over a neutrino spectrum. From Eq. (1) one immediately sees that the solution for $a_{\mu}^{*}(\infty)$ is identical to Eq. (10) with the substitution $a_{e}(-\infty) \rightarrow a_{\mu}^{*}(-\infty)$ and $a_{\mu}(-\infty) \rightarrow-a_{e}^{*}(-\infty)$. Eqs. (7), (9), and (10) then yield

$$
P_{\nu_{e}}=\frac{1}{2}+\frac{1}{2} \cos 2 \theta_{v} \cos 2 \theta_{i}\left(1-2 P_{\text {hop }}\right)
$$

where

$$
P_{\text {hop }}^{\operatorname{lin}}=e^{-\pi \gamma_{c / 2}}
$$

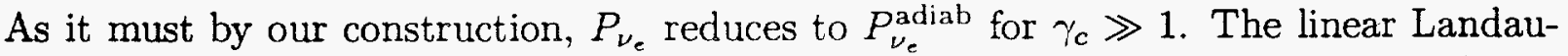
Zener asymptotic hopping probability $P_{\text {hop }}^{\text {lin }}=e^{-\pi \gamma_{c / 2}}$ was derived by Haxton ${ }^{[1]}$ and independently by Parke ${ }^{[2]}$, who married this approximation to the adiabatic one to get Eq. (11a).

The corresponding exponential solution is somewhat more complicated ${ }^{[11]}$,

$$
\begin{aligned}
& a_{e}(\infty)=a_{e}(-\infty) e^{i \delta \epsilon_{i} \cos 2 \theta_{v}} \times \\
& \quad\left[\frac{\Gamma(-i \delta)}{\Gamma\left(-i \frac{\delta}{2}\left(1-\cos 2 \theta_{v}\right)\right)} e^{\frac{\pi \delta}{4}\left(1+\cos 2 \theta_{v}\right)} e^{i \delta \epsilon_{f}}+\frac{\Gamma(i \delta)}{\Gamma\left(i \frac{\delta}{2}\left(1+\cos 2 \theta_{v}\right)\right)} e^{-\frac{\pi \delta}{4}\left(1-\cos 2 \theta_{v}\right)} e^{-i \delta \epsilon_{f}}\right] \\
& \quad-a_{\mu}(-\infty) e^{-i \delta \epsilon_{i} \cos 2 \theta_{v}} \sin 2 \theta_{v} \times \\
& {\left[\frac{\Gamma(i \delta)}{\left(1-\cos 2 \theta_{v}\right) \Gamma\left(i \frac{\delta}{2}\left(1-\cos 2 \theta_{v}\right)\right)} e^{\frac{\pi \delta}{4}\left(1+\cos 2 \theta_{v}\right)} e^{-i \delta \epsilon_{f}}\right.} \\
& \left.-\frac{\Gamma(-i \delta)}{\left(1+\cos 2 \theta_{v}\right) \Gamma\left(-i \frac{\delta}{2}\left(1+\cos 2 \theta_{v}\right)\right)} e^{-\frac{\pi \delta}{4}\left(1-\cos 2 \theta_{v}\right)} e^{i \delta \epsilon_{f}}\right],
\end{aligned}
$$

where $\rho(x)=\rho_{c} e^{-x / r_{0}}$ (so the crossing point is defined as $\left.x=0\right), \delta=\frac{r_{0} \delta m^{2}}{2 E}=$ $\frac{\cos 2 \theta_{v}}{\sin ^{2} 2 \theta_{v}} \gamma_{c}, \epsilon_{f}=\lim _{x \rightarrow \infty} \ln \sqrt{f(x)}$, and $\epsilon_{i}=\lim _{x \rightarrow-\infty}\left(\ln \sqrt{f(x)}-\frac{f(x)}{2 \delta \cos 2 \theta_{v}}\right)$, with $f(x)=$ 
$\sqrt{2} G_{F} r_{0} \rho(x)$. [There are two rapidly varying adiabatic phases, as the integrations are extended to $\pm \infty$, because the density is not symmetric.] The phases again average out in $P_{\nu_{e}}$ when the neutrino-producing region is finite or the neutrino spectrum continuous. From Eqs. (7), (8), and (12) one finds the result of Eq. (11a) but with

$$
P_{\text {hop }}^{\exp }=\frac{e^{-\pi \delta\left(1-\cos 2 \theta_{v}\right)}-e^{-2 \pi \delta}}{1-e^{-2 \pi \delta}}
$$

Exponential descriptions of the transition from adiabatic to nonadiabatic level crossings were discussed by Pizzochero ${ }^{[4]}$, by Toshev ${ }^{[5]}$, and by Petcov ${ }^{[6]}$. Toshev obtained the survival probability for an infinite exponential profile (the limit as $\theta_{i} \rightarrow \pi / 2$ of Eqs. (11a) and (13)), while the resonance-density-independent terms in Petcov's expansion of his finite exponential Landau-Zener result are precisely equivalent to Eqs. (11a) and (13).

The exponential result of Pizzochero

$$
P_{\text {hop }}^{\mathrm{Pizz}}=e^{-\pi \delta\left(1-\cos 2 \theta_{v}\right)}
$$

is widely used but does not correspond to Eq. (13). Note that $\delta(1-\cos 2 \theta)=\gamma_{c} \cos 2 \theta /(1+$ $\cos 2 \theta)$. Thus for small angles $\delta(1-\cos 2 \theta) \rightarrow \gamma_{c} / 2$, and $\delta \rightarrow \gamma_{c} /(2 \theta)^{2}$ becomes large in the nonadiabatic region $\left(\gamma_{c} \sim 1\right)$. Thus Eq. (13) and (14) then both reduce to the linear result of Eq. (11b). However, as we will argue below, the advantages of the exponential form come in the treatment of large $\theta_{v}$, and in that case $\delta$ and $\gamma_{c}$ are of comparable magnitude. Eq. (14) is then not an accurate approximation to the correct result of Eq. (13).

Eq. (11b) and Eq. (13) provide convenient descriptions of the shape of $P_{\nu_{e}}(E)$ as one crosses from the adiabatic to the nonadiabatic region. Which is better to use in solar calculations? First we note that any (infinite) Landau-Zener approximation will fail if the nonadiabatic region (say $\gamma(x) \lesssim 10$ ) around the crossing $x_{c}$ for the true density profile includes the starting or ending densities $\rho\left(x_{i}\right)$ or $\rho\left(x_{f}\right)$ : our assumption of adiabatic propagation in the unphysical regions is then violated. In fact this problem arises only for $x_{i}$ and only when $x_{i}$ is not at the sun's center. If we consider $x_{i} \sim x_{c}$ and $\gamma_{c} \sim 1$ (this would correspond to small mixing angles and large $\delta m^{2} / 2 E$, with a crossing near the sun's center) and vary $E$, an exact treatment changes $P_{\nu_{e}}(E)$ continuously across $E^{c}$, the neutrino energy where $x_{c}$ and $x_{i}$ coincide, $2 \sqrt{2} E^{c} \rho\left(x_{i}\right)=\delta m^{2} \cos 2 \theta_{v}$. That is, the $P_{\text {hop }}$ evolves continuously as the nonadiabatic interval surround $x_{c}$ is moved through $x_{i}$ by changing $E$. But in the Landau-Zener case, one would use the adiabatic result whenever $E<E^{c}, P_{\text {hop }}=0$ (no level crossing), and Eq. (11b) or Eq. (13) for $E>E^{c}$, thus approximating $P_{\nu_{e}}$ by a step function. Fortunately the transition region in $E$ for small $\theta_{v}$ is very sharp, so the step function approximation, while failing in 
a technical sense, yields excellent results when integrated over an extended neutrinoproducing core or continuous neutrino spectrum.

Eq. (11b) and Eq. (13) are differentiated, however, by another consideration, whether the nonadiabatic regions in these approximate profiles include the physical starting and ending densities, $\rho\left(x_{i}\right)$ and $\rho\left(x_{f}\right)=0$. A particularly clear example is a neutrino produced precisely at $x_{i}=0$ and experiencing a crossing at $x_{c}$ near $x_{i}, \rho\left(x_{c}\right)<\rho\left(x_{i}\right)$. For the true solar density, the nonadiabatic region is centered on $x_{c}$ and confined to physical densities: $\gamma\left(x_{i}\right)=\infty$ because the derivative in Eq. (4) vanishes. But the approximate density profile may not share this attractive property: $\gamma\left(x_{i}\right)_{\text {approx }}$ may not be large, so that the nonadiabatic region for the approximate profile may extend into unphysical densities. This contradicts the assumption of adiabatic propagation in unphysical densities that we made in extending the finite Landau-Zener integration to $x=-\infty$. Fortunately, as $\sin ^{2} 2 \theta_{v}$ is typically $\lesssim 10^{-3}$ for nonadiabatic crossings near the solar core, the nonadiabatic region of Eq. (4) is extraordinarily narrow whether one employs the exact or an approximate density. Thus the range of $x_{c}$ where this problem arises is very small, and can be safely ignored in numerical calculations, regardless of whether the exponential or linear Landau-Zener approximations is used.

However, the analogous problem for nonadiabatic crossings near the solar surface is more troublesome: the linear Landau-Zener treatment requires an additional extension to unphysical densities as $x \rightarrow+\infty$. It does not share an important property of the exponential treatment, that $\gamma(x) \rightarrow \infty$ as one approaches the solar surface $(\rho=0)$. That is, it is apparent from Eq. (4) that the nonadiabatic region becomes very broad for large $\theta_{v}$, and often extends into the region of negative densities for a linear potential. If this happens, there is no reason to expect the linear Landau-Zener result to be accurate. This distinguishes the linear and exponential approximations, and suggests that the exponential is the preferred choice for large $\theta_{v}$.

A comparison of the Landau-Zener approximations to exact calculations is made in Fig. 2. The exact calculations were performed by integrating Eq. (1) in the vicinity of the crossing point by an adaptive algorithm that controls local and accumulated errors, and then matching to the adiabatic result in regions where this result is essentially exact $(\gamma(x) \gtrsim 30)$. The form of Eq. (1) used in this integration is that of Eqs. (21) and (22) of Ref. [10]: we express Eq. (1) in terms of two solutions that evolve locally with the correct adiabatic phases, $\pm i \int_{0}^{x} \lambda\left(x^{\prime}\right) d x^{\prime}$. This, of course, removes the most rapidly varying components from Eq. (1), simplifying the numerical integration. An analytic fit ${ }^{[12]}$, continuous and with continuous derivatives, to the solar electron density is used. This function was determined by fitting the standard solar model densities tabulated by Bahcall ${ }^{[13]}$, and is generally accurate to $\lesssim 1 \%$. The neutrino is assumed to be produced 
exactly at the solar center.

The exact integrations determine the surface amplitudes $a_{L}\left(x_{f}\right)$ and $a_{H}\left(x_{f}\right)$. The relative phasing of these amplitudes depends on the accumulated adiabatic phases, and thus oscillates rapidly with variations in the neutrino production point or energy. Thus the physically relevant quantity in any realistic calculation that integrates over the spatial extent of the neutrino-producing solar core or over the neutrino spectrum is the $\nu_{e}$ survival probability

$$
\begin{aligned}
P_{\nu_{e}} & =\left|a_{L}\left(x_{f}\right)\right|^{2}\left|\left\langle\nu_{e} \mid \nu_{L}\left(x_{f}\right)\right\rangle\right|^{2}+\left|a_{H}\left(x_{f}\right)\right|^{2}\left|\left\langle\nu_{e} \mid \nu_{H}\left(x_{f}\right)\right\rangle\right|^{2} \\
& =\frac{1}{2}+\frac{1}{2} \cos 2 \theta_{v}\left(1-2\left|a_{H}\left(x_{f}\right)\right|^{2}\right) .
\end{aligned}
$$

Clearly this is the appropriate quantity to compare to the various analytic results, since a similar averaging of phases was done in those derivations. $P_{\nu_{e}}$ is also the neutrino survival probability a long distance from the sun, as $a_{L}(x)$ and $a_{H}(x)$ do not evolve in vacuum apart from the associated vacuum mass eigenstate phases.

In accordance with our qualitative arguments, the various Landau-Zener results are virtually indistinguishable for small $\sin ^{2} 2 \theta$, when the nonadiabatic region is a very narrow interval surrounding the crossing point. The agreement with the exact calculations is also excellent. But for $\sin ^{2} 2 \theta \gtrsim 0.05$ discernible differences appear for small $\delta \mathrm{m}^{2} / E$ corresponding to nonadiabatic crossings occuring in the outer layers of the sun. While the exponential Landau-Zener continues to agree with the exact calculations to an accuracy $\lesssim 2 \%$, the linear form overestimates the hopping probability: the linear profile underestimates $\gamma(x)$ for $x>x_{c}$, and the nonadiabatic region has become suffciently broad for large $\sin ^{2} 2 \theta$ that the accumulated error is apparent. It is also clear from Fig. 2d that Pizzochero's form of the exponential Landau-Zener probability fails: it agrees more closely with the linear result than the more correct exponential result, and among the three Landau-Zener formulas provides the poorest description of the exact results for small $\delta m^{2} / E$.

In the case of a neutrino produced on the far side of the sun at a density $\rho\left(x_{i}\right)<$ $\rho\left(x_{c}\right)$, two crossings are encountered as the neutrino travels toward the earth. The derivation of the survival probability in the Landau-Zener approximation can be done is precise analogy with the single crossing case: two finite wedges with the proper slope and density at the crossing point represent the propagation of the neutrino to the central point of maximum density, and then the propagation from that point to the solar surface. The amplitudes for each section can be obtained by extending the wedges to infinity under the assumption of adiabatic propagation in the unphysical regions. The analogs of Eqs. (10) and (12) for $\dot{\rho}>0$ are then needed for the first wedge. The final 
result is

$$
P_{\nu_{e}}^{\text {double crossing }}=\frac{1}{2}+\frac{1}{2} \cos 2 \theta_{v} \cos 2 \theta_{i}\left(1-4 P_{\text {hop }}\left(1-P_{\text {hop }}\right)\right)
$$

The derivation of Eq. (16) requires an averaging over the relative phases accumulated between the first and second crossings, in addition to the averaging of the asymptotic phases going into the first crossing and leaving the second. Such averaging is again physically motivated because all of these phases vary rapidly with changes in the neutrino energy or production point within the sun.

In Fig. 3 we compare results from Eq. (16) with exact integrations. The neutrino is produced at a radius $x_{i}=0.3 R_{\odot}$ on the far side of the sun, and thus passes through the solar center on its way to the earth. The magnitudes $\left|a_{L}\left(x_{f}\right)\right|$ and $\left|a_{H}\left(x_{f}\right)\right|$ now depend on the exact path and energy (there is interference between the two hopping amplitudes), so one must average the exact $P_{\nu_{e}}$ before a meaningful comparison with Eq. (16) can be made. We do so by making small variations in $x_{i}$ in a symmetric interval about $0.3 R_{\odot}$, fitting the resulting variations in $P_{\nu_{e}}$ by a function of the form $\mathrm{A}+\mathrm{B} \cos \left(\omega x_{i}+\phi\right)$, and then equating $\bar{P}_{\nu_{e}}=\mathrm{A}$. This procedure yields a very nice representation of the numerical results. The comparisons in Fig. 3 are very much like those of Fig. 2, with discernible differences between the calculations arising only for large mixing angles. Fig. $3 \mathrm{~d}\left(\sin ^{2} 2 \theta_{v}=0.5\right)$ again shows that the exponential form is somewhat more accurate for small $\delta m^{2} / E$.

In conclusion, we have attempted to provide a clear derivation of Landau-Zener formulae for solar neutrino oscillation probabilities to illustrate the conditions for their validity. The linear and exponential forms and exact calculations agree very well except when $\delta m^{2} / E$ is small and $\sin ^{2} 2 \theta$ is large. This exception corresponds to nonadiabatic crossing occuring in the outer layers of the sun where $\gamma(x) \lesssim 10$ over a substantial region (typically $\Delta x \sim 0.1 R_{\odot}$ ). The exponential formula remains quite accurate, while deviations of the linear formula from the exact results exceed $10 \%$ for $\sin ^{2} 2 \theta=0.5$ and $\delta m^{2} / E=10^{-9} \mathrm{eV}^{2} / \mathrm{MeV}$. The exponential formula of Pizzochero yields a fit that is similar to the linear result, but slightly poorer. This modest failure of the linear profile is readily understood as resulting from its tendency to underestimate $\gamma(x)$ for $x>x_{c}$, resulting in an extension of the nonadiabatic region into unphysical densities (beyond $\rho=0$ ). Thus it overestimates the hopping probability for large $\sin ^{2} 2 \theta$ and small $\delta m^{2} / E$.

This work was supported in part by the U.S. Department of Energy and by NASA under grant \#NAGW2523. Marcus Bruggen thanks the Institute for Nuclear Theory for a summer fellowship for undergraduate research. 


\section{References}

[1] W.C. Haxton, Phys. Rev. Lett. $\underline{57}$ (1986) 1271.

[2] S.J. Parke, Phys. Rev. Lett. $\underline{57}$ (1986) 1275.

[3] D. Nötzold, Phys. Rev. D $\underline{36}$ (1987) 1625.

[4] P. Pizzochero, Phys. Rev. D $\underline{36}$ (1987) 2293.

[5] S. Toshev, Phys. Lett. B 196 (1987) 170. Note, however, that Eqs. (10) and (13) in this paper are correct for an exponentially increasing density.

[6] S.T. Petcov, Phys. Lett. B 200 (1988) 373.

[7] S.P, Mikheyev and A. Yu Smirnov, Yad. Fiz. 42 (1985) 1441 [Sov. J. Nucl. Phys. $\underline{42}$ (1985) 913] and Nuovo Cimento $\underline{9}$ C (1986) 17; L. Wolfenstein, Phys. Rev. D. $\underline{17}$ (1987) 2369 and D $\underline{20}$ (1979) 2634.

[8] H. Bethe, Phys. Rev. Lett. 56 (1986) 1305; also see A. Messiah, in '86 Massive Neutrinos in Astrophysics and in Particle Physics, ed. O. Fackler and J. Tran Thanh Van (Editions Frontières, Gif-sur-Yvette, 1986) p. 373.

[9] W.C. Haxton, Nucl. Phys. A570 (1994) 125C.

[10] W.C. Haxton Phys. Rev. D $\underline{35}$ (1987) 2352.

[11] L.D. Landau, Phys. Z. Sowjetunion 2 (1932) 46; C. Zener, Proc. R. Soc. London A 137 (1932) 696; E.C.G. Stueckelberg, Helv. Phys. Acta $\underline{5}$ (1932) 369.

[12] W.C. Haxton, unpublished.

[13] J.N. Bahcall, Neutrino Astrophysics, Cambridge University Press; J.N. Bahcall and M.H. Pinsonneault, Rev. Mod. Phys. $\underline{64}$ (1992) 885. 


\section{Figure Captions}

Figure 1. The solid line in the top figure shows, for a neutrino produced at the sun's center with $\sin ^{2} 2 \theta=0.005$ and $\delta m^{2} / E=10^{-6} \mathrm{eV}^{2} / \mathrm{MeV}$, that the region of nonadiabatic propagation, defined here as $\gamma(x) \lesssim 10$, is confined to a narrow region about the crossing point $x_{c}$. For these parameters the crossing point is $x_{c}=0.34 R_{\odot}$ and $\gamma_{c}=0.78$, so that the transition is near the boundary that separates the adiabatic and nonadiabatic regions in the $\delta m^{2} / E-\sin ^{2} 2 \theta$ plane (see Ref. [10]). In the lower figure, the solid lines represent the solar density $\rho(x)$ and a linear approximation to $\rho(x)$ that has the correct initial and final densities and the correct slope at $x_{c}$. By extending the wedge to $\pm \infty$ (dotted lines and arrows) and assuming adiabatic propagation in the regions of unphysical density, one obtains the simple LandauZener result of Eq. (11).

Figure 2. The electron neutrino survival probability is given as a function of $\delta m^{2} / E$ for a neutrino produced at the sun's center. The solid dots are the results of numerical integrations. The solid, dotted, and dash-dotted lines are the linear, exponential, and Pizzochero-exponential Landau-Zener results. The dashed line is the adiabatic result.

Figure 3. As in Fig. 2, only for a neutrino produced at $x_{i}=0.3 R_{\odot}$ that passes back through the sun's center. The exact (numerically integrated) results were averaged over a narrow neutrino production region centered on $x_{i}$, as discussed in the text. 

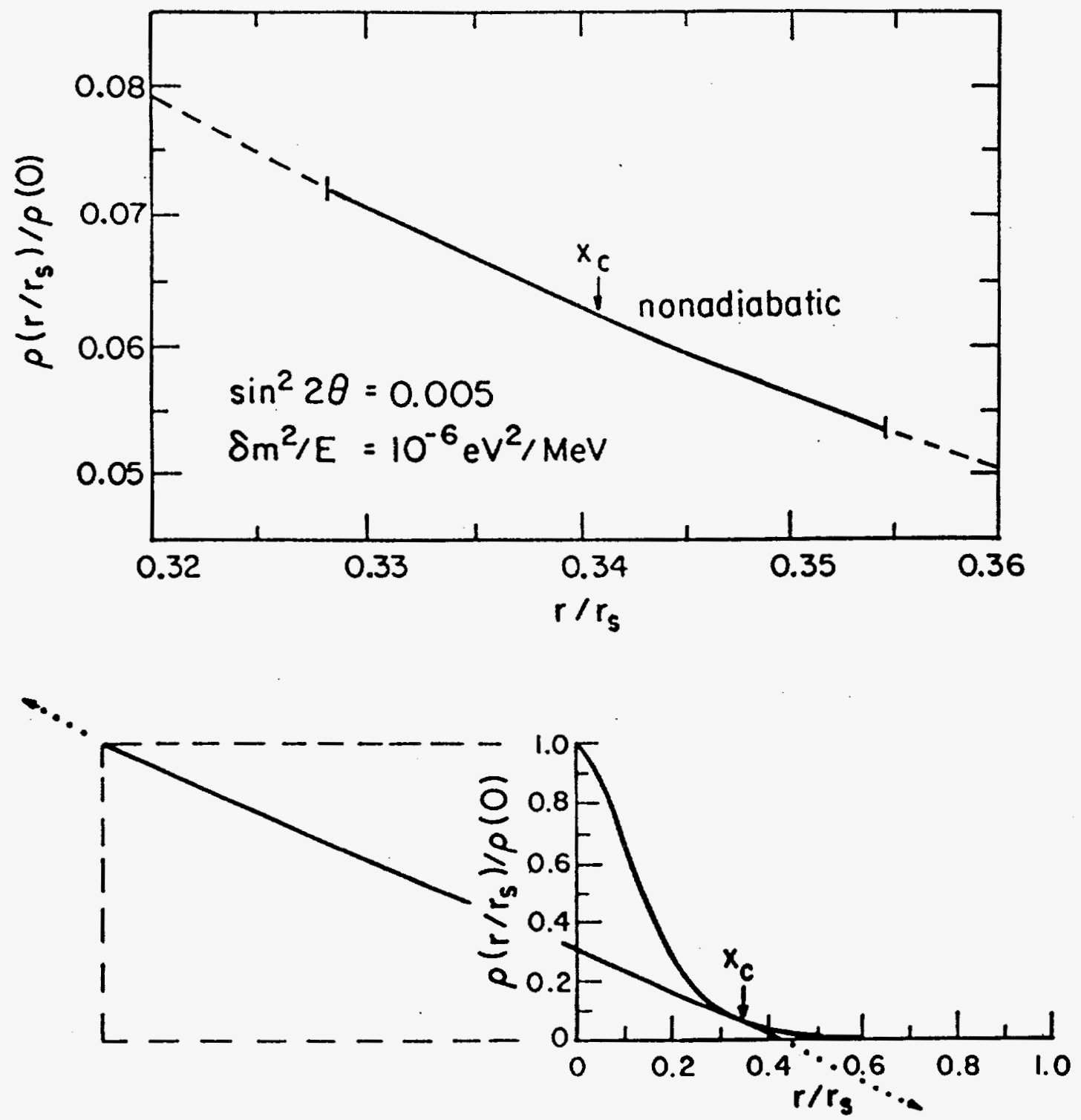

Fig. 1 


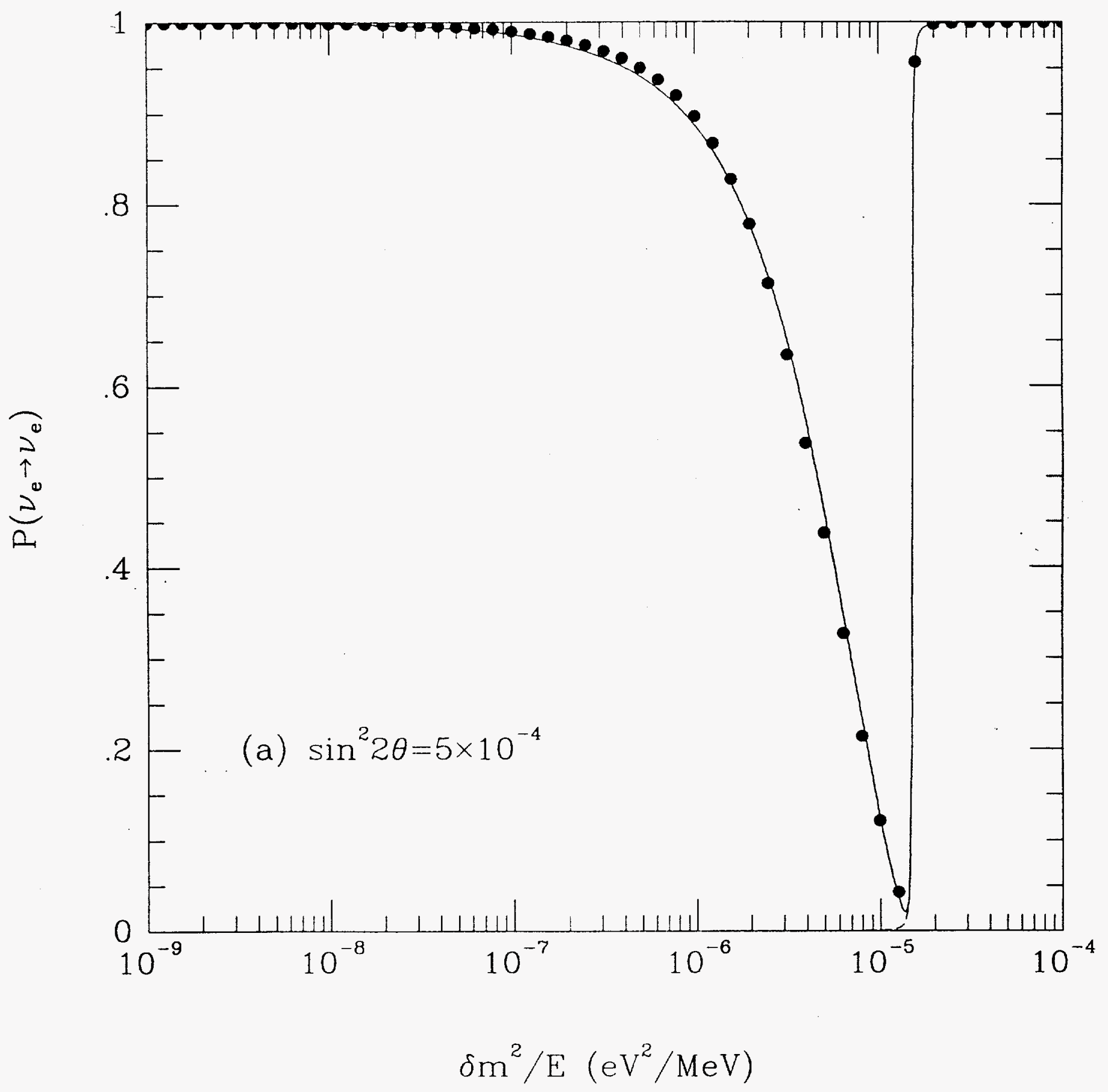

Fig. 2a 


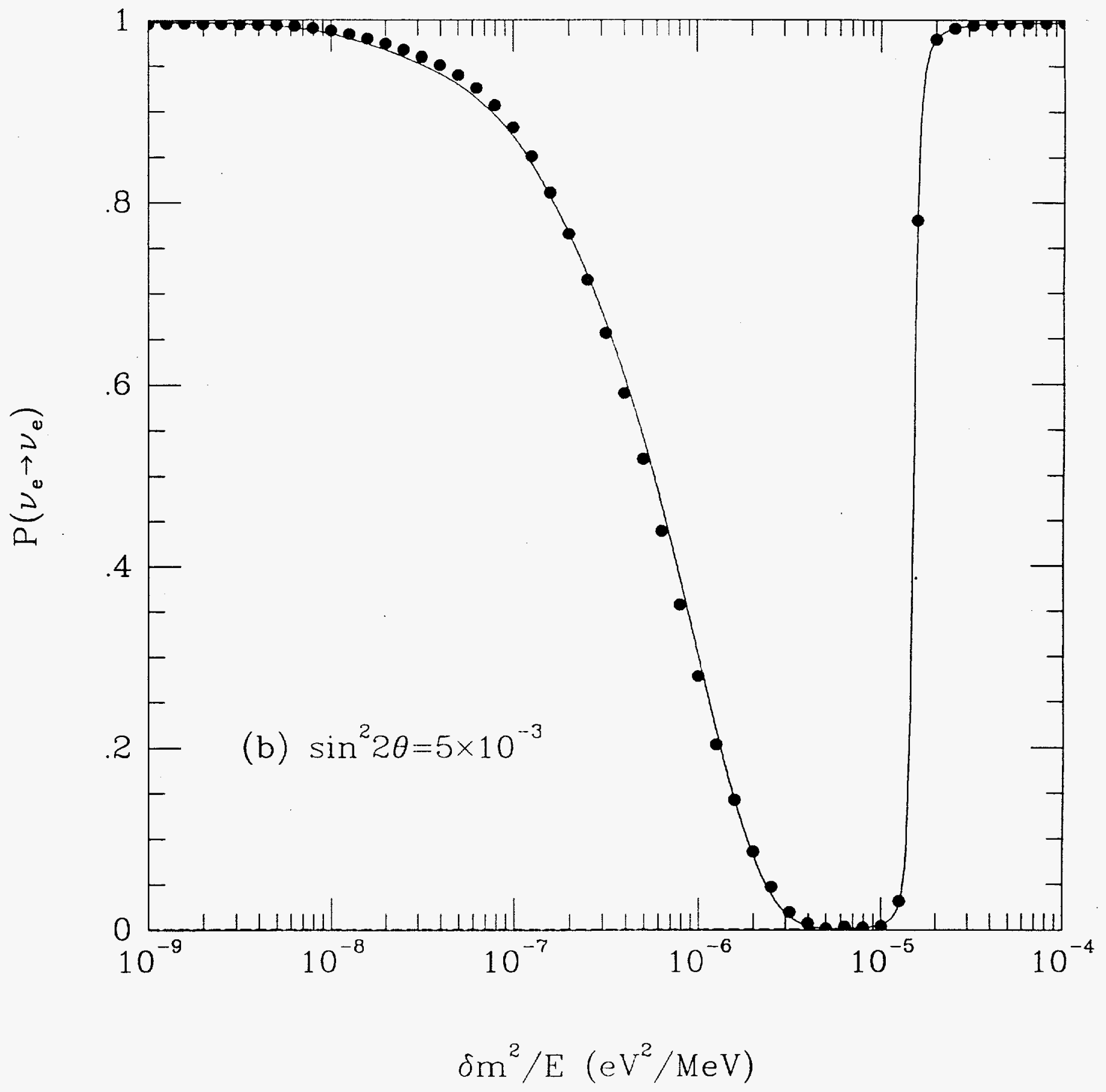

Fig. 2b 


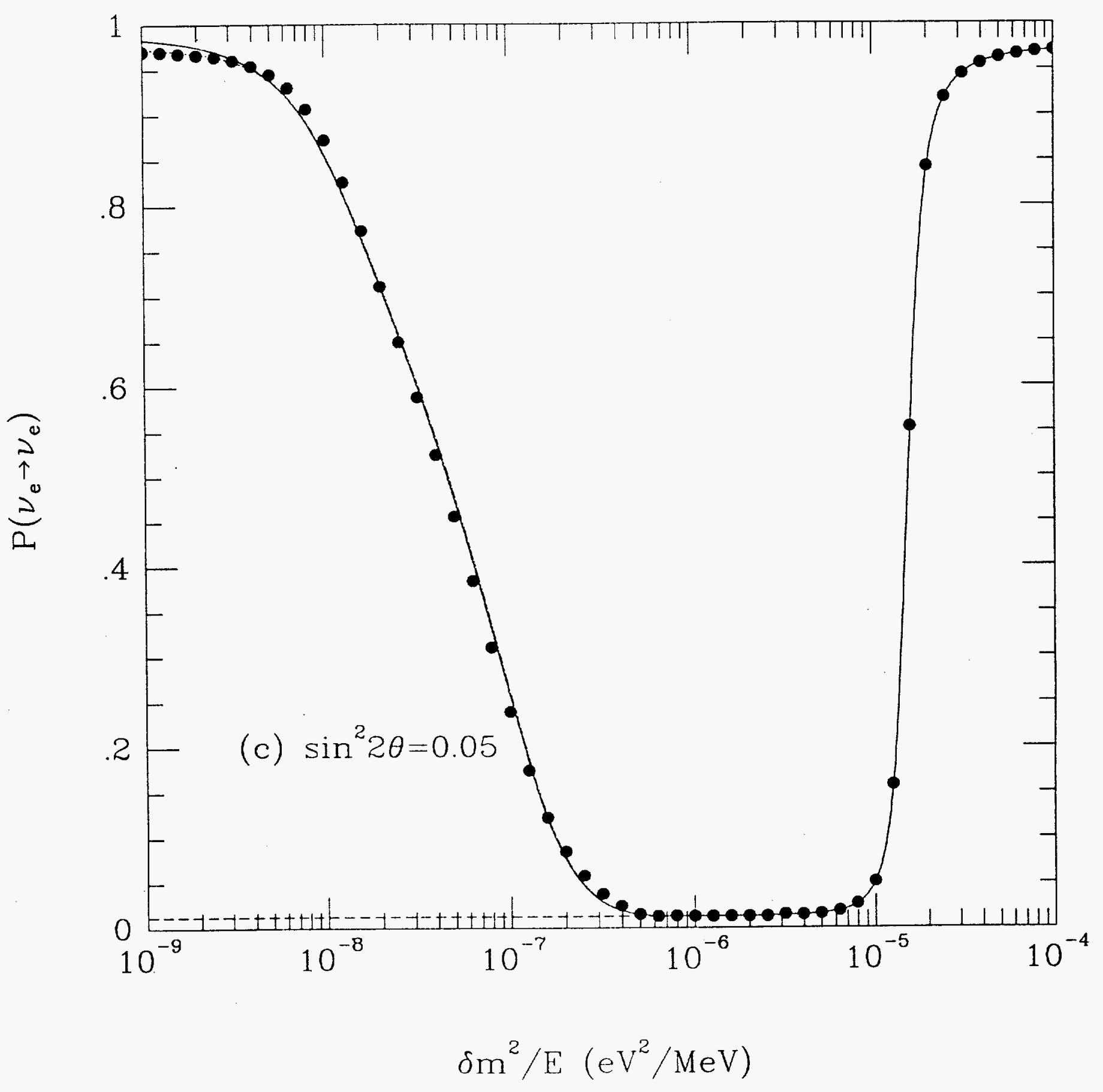

Fig. 2c 


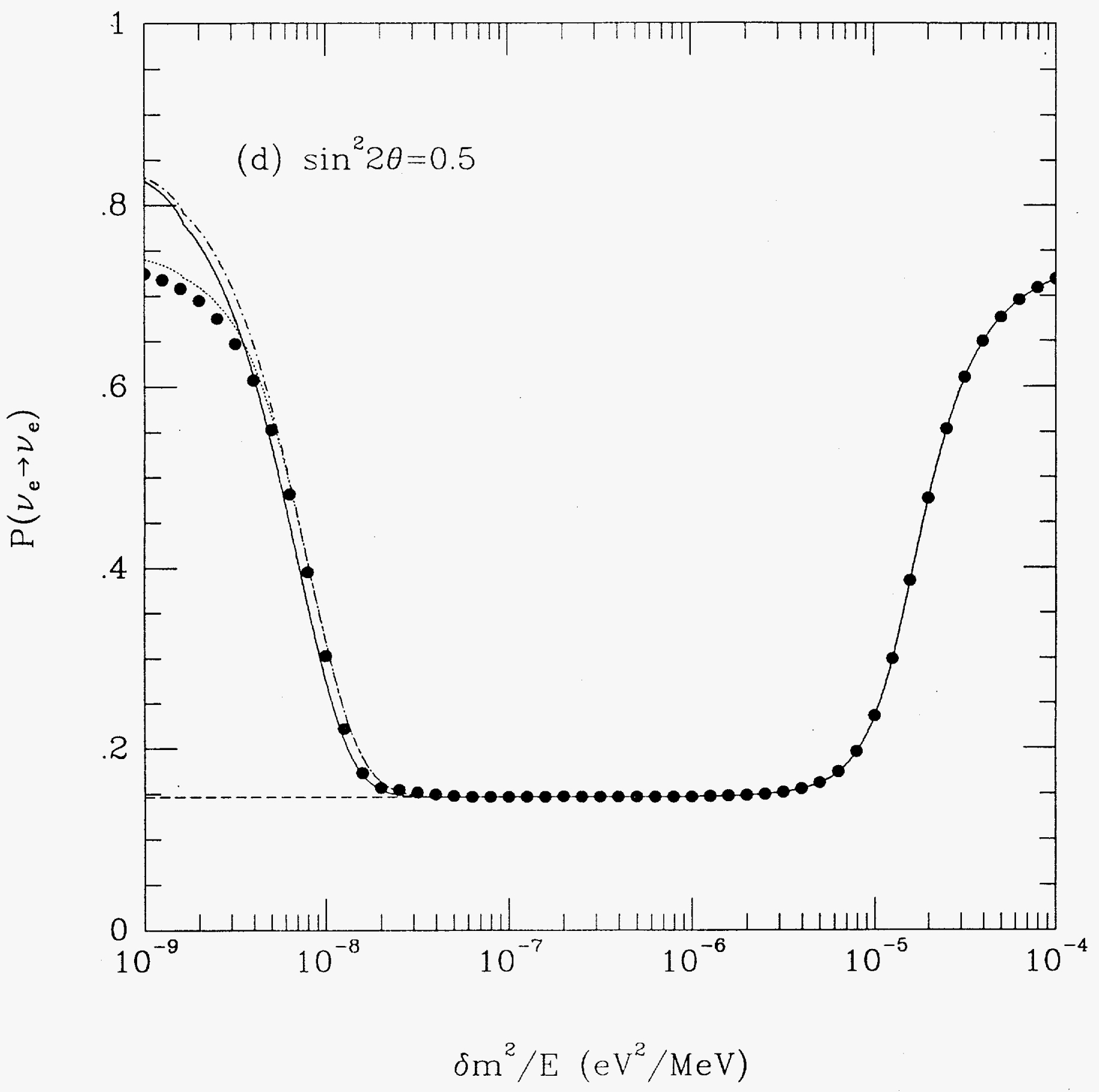

Fig. 2d 


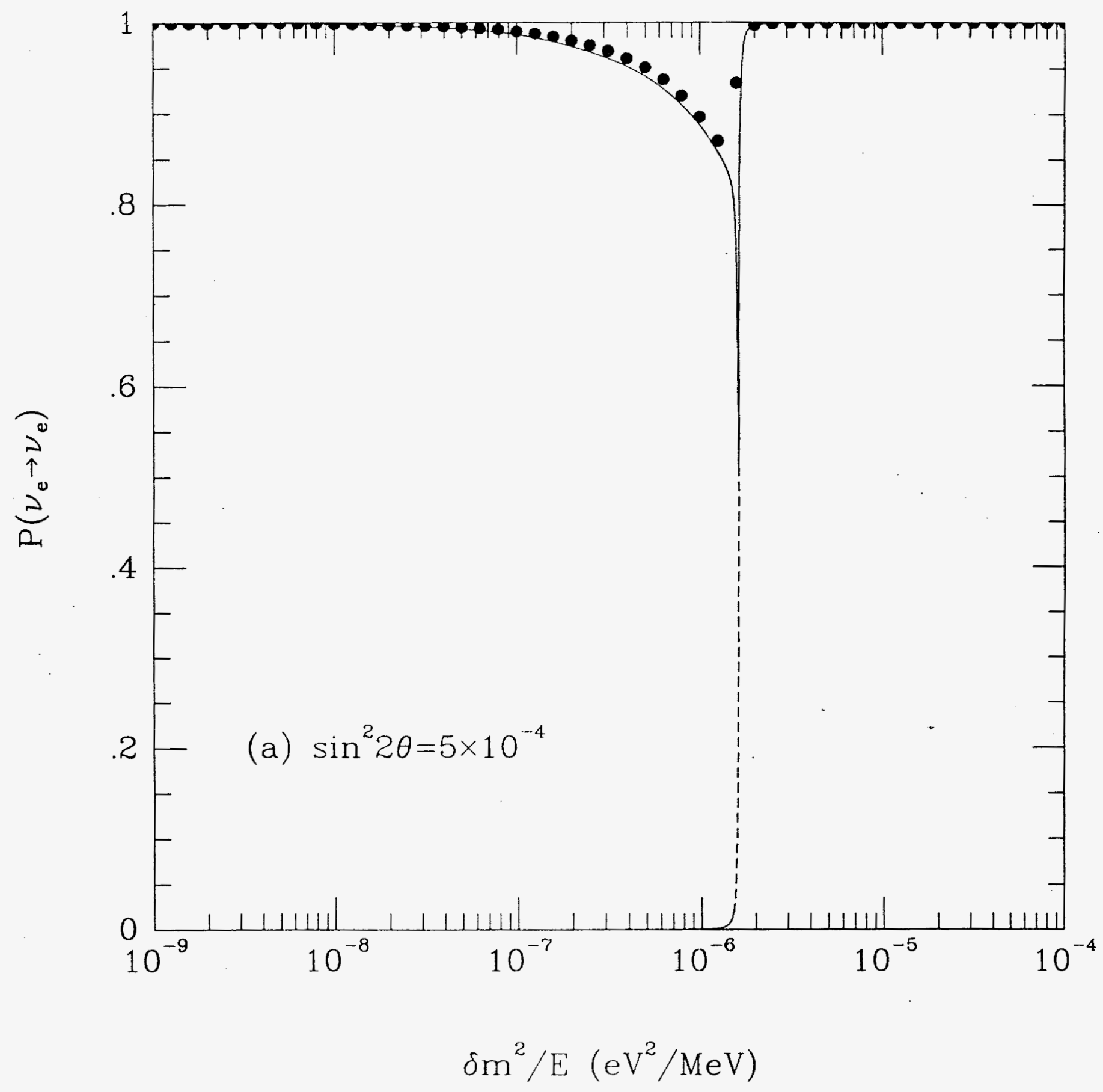

Fig. 3a 


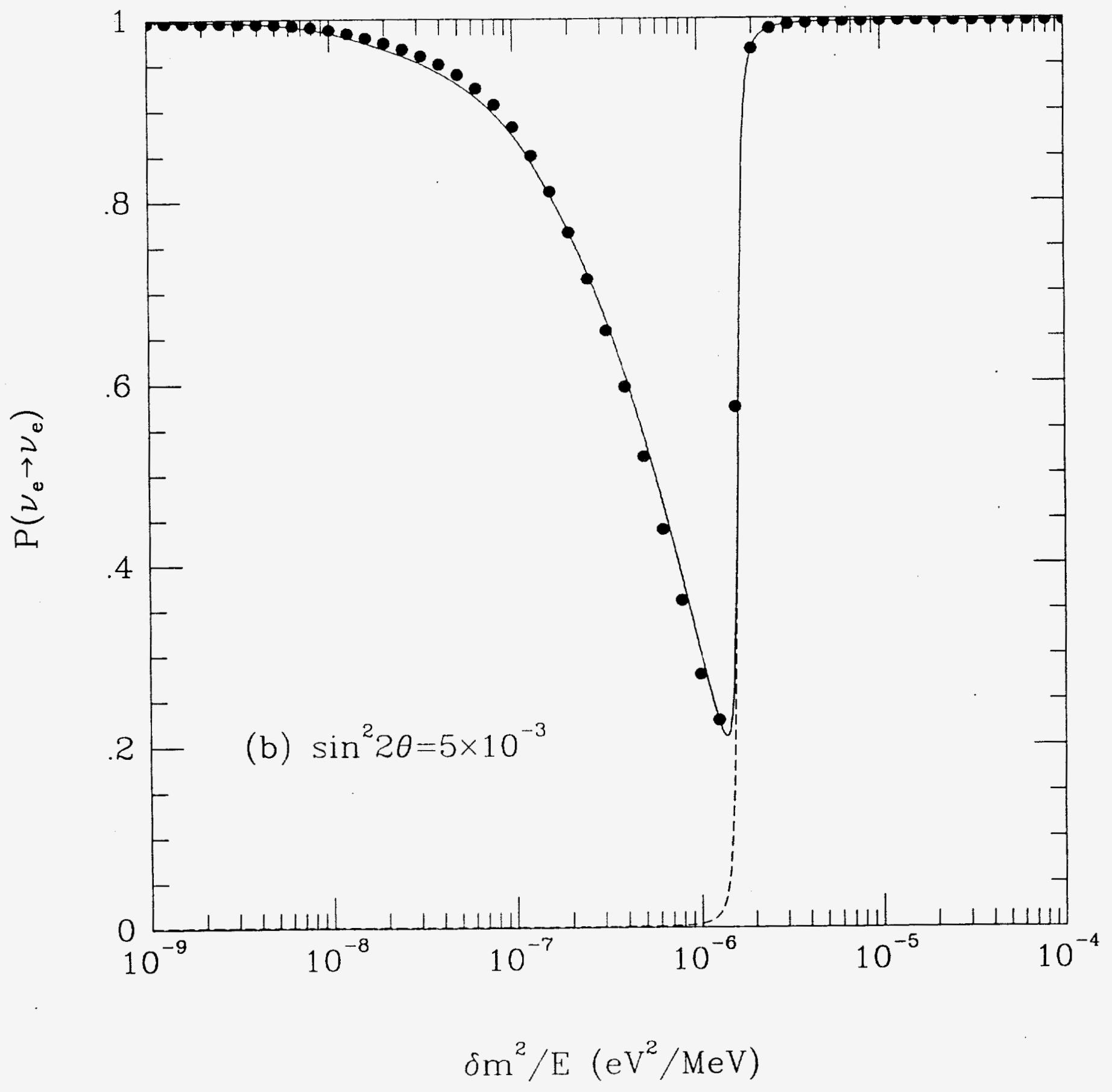

Fig. 3b 


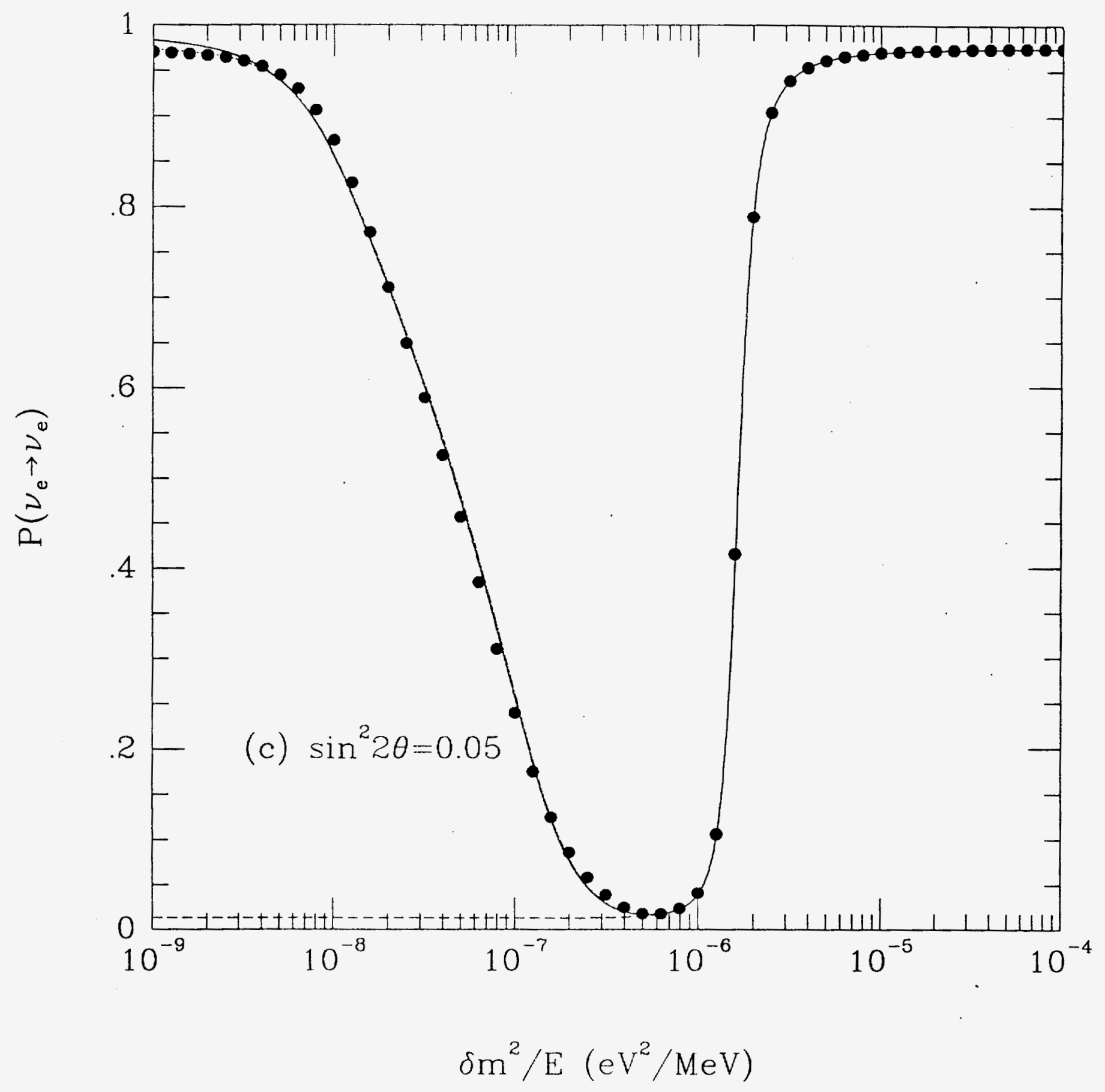

Fig. 3c 


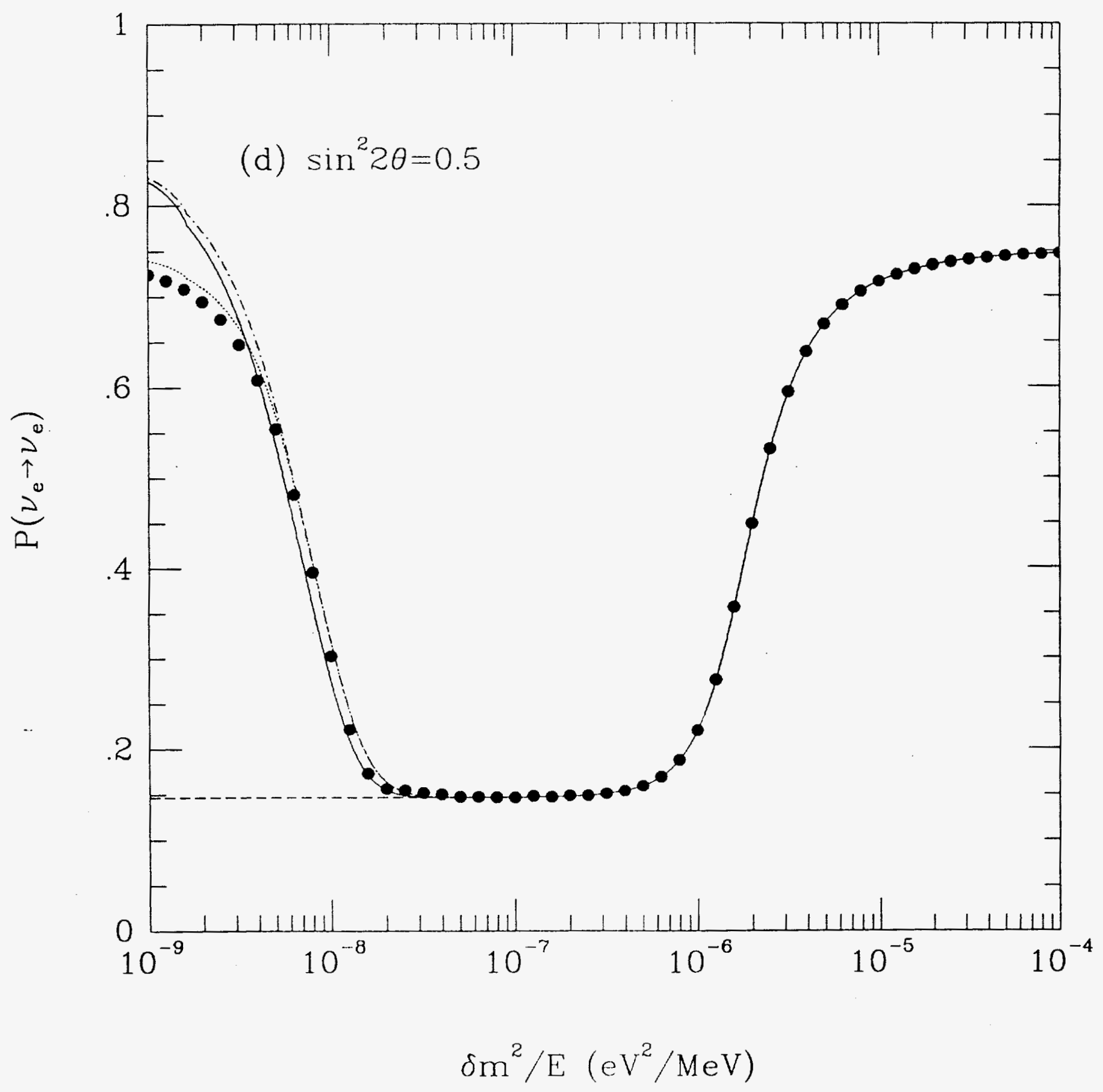

Fig. 3d 